\title{
Dealing with Predictive-but-Unpredictable Attributes in Noisy Data Sources
}

\author{
Ying Yang, Xindong Wu, and Xingquan Zhu \\ Department of Computer Science, University of Vermont, Burlington VT 05405, USA \\ \{yyang, xwu , xqzhu\}@cs.uvm.edu
}

\begin{abstract}
Attribute noise can affect classification learning. Previous work in handling attribute noise has focused on those predictable attributes that can be predicted by the class and other attributes. However, attributes can often be predictive but unpredictable. Being predictive, they are essential to classification learning and it is important to handle their noise. Being unpredictable, they require strategies different from those of predictable attributes. This paper presents a study on identifying, cleansing and measuring noise for predictive-but-unpredictable attributes. New strategies are accordingly proposed. Both theoretical analysis and empirical evidence suggest that these strategies are more effective and more efficient than previous alternatives.
\end{abstract}

\section{Introduction}

Real-world data are seldom as perfect as we would like them to be. Except in the most structured environment, it is almost inevitable that data contain errors from a variety of corrupting processes, such as acquisition, transmission and transcription $[11,13]$. The corrupted data, namely noise, usually have adverse impact on interpretations of the data, models created from the data, and decisions made based on the data [7]. Since a manual process is laborious, time consuming and itself prone to errors, effective and efficient approaches that automate noise handling are necessary [8].

This paper handles noise in the context of classification learning, which plays an active role in machine learning. In classification learning, data are composed of instances. Each instance is expressed by a vector of attribute values and a class label. We further differentiate attributes into three types: unpredictive, predictive-and-predictable and predictive-but-unpredictable. Unpredictive attributes are futile for or irrelevant to predicting the class. They can be discarded by feature selection methods prior to the learning. Predictive attributes are useful for predicting the class, among which predictable ones can be predicted by the class and other attributes while unpredictable ones cannot. We argue that since predictive attributes contribute to classification learning, it is important to handle their noise. However, because predictable attributes and unpredictable attributes have different natures, they require different strategies for noise handling. For convenience of expression, an 'attribute' throughout this paper implies a predictive attribute unless otherwise mentioned. 
Generally, there are two types of noise, attribute noise and class noise. Most previous efforts are engaged in handling class noise, such as for contradictory instances (the same instances with different class labels) or misclassifications (instances labeled with a wrong class). Various achievements have been reported [2$6,14,15]$. In comparison, much less attention has been paid to attribute noise. Ironically, attribute noise tends to happen more often in the real world. For example, if noise comes from entry mistakes, it is very likely that the class has fewer errors since the people involved know that it is the 'important' value and pay more attention to it [11].

Among few publications on handling attribute noise, an important contribution is LENS [7]. It aims at presenting a 'complete' understanding that helps identify, correct or prevent noise by modelling the generation of clean data, the generation of noise and the process of corruption, under the assumption that the noise generation and the corruption process have learnable underlying structures. If the assumption does not hold, the authors indicate that LENS has limited utility. Different from LENS, another often-cited achievement, polishing [12], deals with attribute noise without knowing its underlying structures. As we will detail later, polishing excels in handling noise for predictable attributes. A predictable attribute is one that can be predicted by the class and other attributes. We suggest that attributes in real-world applications can frequently be otherwise. It is because normally attributes are collected as long as they contribute to predicting the class. Whether or not one attribute itself can be predicted is often not a concern.

Hence, with all due respect to previous achievements, we suggest that the picture of handling attribute noise is incomplete. It is an open question how to address unpredictable attributes when their underlying noise structures are unavailable. These understandings motivate us to explore appropriate strategies that identify, cleanse and measure noise for predictive-but-unpredictable attributes. In particular, Section 2 introduces the background knowledge. Section 3 proposes sifting to identify noise. Section 4 discusses deletion, uni-substitution and multi-substitution to cleanse the identified noise. Section 5 studies literal equivalence and conceptual equivalence to measure noise. Section 6 empirically evaluates our new strategies. Section 7 gives a conclusion and suggests further research topics.

\section{Background Knowledge: Polishing}

Polishing [12] is an effective approach to handling noise for predictable attributes. When identifying noise for an attribute $A$, polishing swaps $A$ with the original class, and uses cross validation to predict $A$ 's value for each instance. Those values that are mis-predicted are identified as noise candidates. It then replaces each noise candidate by its predicted value. If the modified instance is supported by a group of classifiers learned from the original data, the modification is retained. Otherwise, it is discarded. Polishing assumes that since one can predict the class by attribute values, one can turn the process around and use the class 
Table 1. Prediction accuracies of classes and attributes in polishing's data. For instance, mushroom has $100.0 \%$ in the 'Class' column indicating that the prediction accuracy for its class is $100.0 \%$. Mushroom has 10 in the column [90\%,100\%] of 'Attributes' indicating that it has 10 attributes whose prediction accuracies fall into [90\%,100\%].

\begin{tabular}{l|r|cccc}
\hline Dataset & Class & \multicolumn{4}{|c}{ Attributes } \\
& & {$[90 \%, 100 \%]$} & {$[70 \%, 90 \%)$} & {$[50 \%, 70 \%)$} & {$[0 \%, 50 \%)$} \\
\hline mushroom & $100.0 \%$ & 10 & 4 & 5 & 3 \\
soybean & $91.5 \%$ & 27 & 3 & 3 & 2 \\
led-24 & $100.0 \%$ & 7 & 0 & 17 & 0 \\
vote & $96.3 \%$ & 3 & 10 & 3 & 0 \\
audiology & $77.9 \%$ & 58 & 9 & 0 & 2 \\
promoters & $81.1 \%$ & 0 & 0 & 3 & 54 \\
\hline
\end{tabular}

together with some attributes to predict another attribute's values as long as this attribute is predictive.

However, this 'turn around' can be less justifiable when unpredictable attributes are involved. For example, the often-cited monk's problems from the UCI data repository [1] have 6 attributes $A_{1}, \ldots, A_{6}$ and a binary class. One underlying concept is $\left(A_{4}=1\right.$ and $\left.A_{5}=3\right)$ or $\left(A_{2} \neq 3\right.$ and $\left.A_{5} \neq 4\right) \Rightarrow C=1$; otherwise $C=0$. Accordingly $A_{5}$ is a predictive attribute. Although the class can be predicted with $100 \%$ accuracy by C4.5trees [9] using 10-fold cross validation, the prediction accuracy for $A_{5}$ is only $35 \%$ because it has multiple values mapped to a single class. Directed by such a low accuracy, the noise identification has a strong potential to be unreliable. More datasets involving predictive-butunpredictable attributes will be shown in Section 6 .

Polishing has reported favorable experimental results. Nonetheless, they apply to predictable attributes. For each of polishing's datasets, Table 1 summarizes prediction accuracies for its class and attributes ${ }^{1}$. In all datasets except 'promoters', highly predictable attributes dominate the data ${ }^{2}$. In many cases, attributes are even more predictable than the original class. The 'promoters' data, which are not dominated by predictable attributes, produce a less favorable result for polishing.

These observations by no means devalue polishing's key contribution to handling predictable attributes. Nonetheless they raise the concern of polishing's suitability for unpredictable attributes and inspire our further study.

\section{Identifying Noise}

We propose a sifting approach to identifying noise for unpredictable attributes. For a predictable attribute, there exists a value whose probability given an in-

\footnotetext{
${ }^{1}$ We summarize them because many datasets have too many attributes to be listed individually. For instance, audiology has 69 attributes. Each accuracy results from C4.5trees using 10-fold cross validation, as polishing did.

${ }^{2}$ For the 'vote' dataset, although 17 attributes fall in [50\%,70\%], these attributes are randomly-added unpredictive attributes.
} 
stance is high enough to dominate alternative values. For an unpredictable attribute, there is often no such dominating value. Instead, multiple values may be valid given an instance.

To identify noise for an attribute, polishing predicts what the clean value is and identify other values suspicious. This strategy is less appropriate if the attribute is unpredictable. For example, we have instances to represent apples and berries. One attribute is color. Apples can be green, yellow or red. Berries can be blue, black or red. If green apples happen to have a slightly higher occurrence, polishing will identify valid instances like $\langle\cdots$, yellow, apple, $\cdots\rangle$ and $<\cdots$, red, apple, $\cdots\rangle$ suspicious since the predicted color of an apple is green.

Neither is it adequate to individually identify noise for each attribute, where an attribute is separated from the others, swaped with the class and predicted by other possibly noisy attributes. Suppose an instance to be $<$ $\cdots$, black, apple, $\cdots>$. When the attribute 'color' is under identification, its value 'black' will be identified suspicious since it should be 'green' given 'apple'. Meanwhile, when the attribute 'fruit' is under identification, its value 'apple' will be identified suspicious since it should be 'berry' given 'black'. Thus both values are identified. However, it is very likely that only one is real noise. The original instance can be a 'black berry' or a 'green apple'. This compounded suspicion is caused by identifying noise according to noisy evidence.

Accordingly sifting evaluates whether the pattern presented by a whole instance is suspicious instead of judging isolated values. It identifies an instance suspicious only when this instance does not satisfy any pattern that is learned with certain confidence from the data. We name this strategy 'sifting' because it takes the set of learned patterns as a sifter to sift instances. Instances that match any pattern may go through while the remaining are identified suspicious. By this means, an unpredictable attribute is not forced to comply with a single value. Instead, its multiple valid values can be allowed. For instance, colors of green, yellow and red are all allowable for an apple since we can learn those patterns. A black apple will be identified suspicious since its pattern is very unlikely, and this anomaly is efficiently identified in one go.

Algorithm 1 shows an implementation of sifting. Please be noted that we are dealing with situations where the underlying structure of noise (if there is any at all) is not available. This implies that if there is any learnable pattern, it reflects the knowledge of clean data. Furthermore, the learned patterns are in terms of classification rules (with the class, but not any attribute, on the right hand side).

We expect sifting to obtain a subset of instances with a high concentration of noise. We then forward this subset to the next process: cleansing.

\section{Cleansing Noise}

One should be very cautious when coming to cleanse an instance. For example, the data can be completely noise-free but an inappropriate rule learner is employed. Hence, we suggest that noise cleansing is conducted only when the data's 


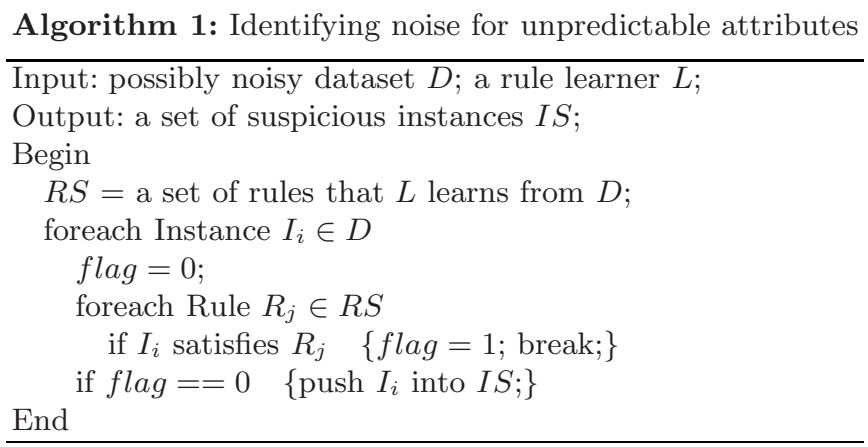

genuine concept is learnable; practically, only when a high prediction accuracy is achievable.

For predictive attributes, it is sensible to only permit changes towards the predicted values, as polishing does. For unpredictable attributes, usually no value can be predicted with such a high accuracy as to exclude alternative values. Accordingly, we study three cleansing approaches, deletion, uni-substitution and multi-substitution.

As delineated in Algorithm 2, deletion simply deletes all identified noisy instances. At first sight, it most likely causes information loss. Nevertheless, this intuition needs to be verified. Uni-substitution cleanses an instance by the rule that minimizes the number of value changes. If there are multiple such rules, uni-substitution filters them by their quality indicators ${ }^{3}$ and chooses a single one to cleanse the suspicious instance. Hence uni-substitution maintains the original data amount. Multi-substitution is the same as uni-substitution except at the final stage. If finally several rules are still equally qualified, multisubstitution produces multiple cleansed instances, each corresponding to a rule, and substitutes all of them for the suspicious instance. In this way, it may increase the data amount. But it has a merit that retrieves all valid values.

\section{Measuring Noise}

It is important to measure how noisy the corrupted data are if compared against its clean version. Otherwise, it is difficult to evaluate the effectiveness of a noise handling mechanism. A common measurement is literal equivalence $[12,15]$. We believe that it is often of limited utility and propose conceptual equivalence instead.

\subsection{Literal Equivalence}

Suppose $C D$ is a clean dataset and is corrupted into a noisy dataset $N D$. When measuring noisy instances in $N D$, literal equivalence conducts a literal comparison. Two common mechanisms are match-corresponding and match-anyone.

\footnotetext{
${ }^{3}$ Normally the rule learner attaches quality indicators to each rule. For example, we employ C4.5rules that attaches each rule with confidence, coverage and advantage.
} 
into $I_{1}$ as in (b) of Figure 2. Although (b) has significantly lost information of (a), match-anyone still judges the cleansing superb with $100 \%$ success.

(a)

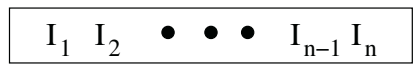

(b)

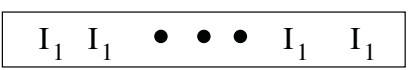

Fig. 2. Match-anyone is insensitive to information loss.

\subsection{Conceptual Equivalence}

Other mechanisms of literal equivalence may well exist. However, all measurements involving literal comparison suffer from a problem that they confine the data's legitimacy to the clean dataset at hand. Usually a dataset is only a sample of the whole population. Instances can legitimately exist in the whole population but do not appear in certain samples. This motivates us to propose conceptual equivalence, which we expect to be more elegant and capable in measuring noise. Suppose a clean dataset $C D$ is corrupted and is then cleansed into a dataset $C D^{\prime}$. Suppose the target concepts learned from $C D$ and $C D^{\prime}$ are Concept $_{C D}$ and Concept $_{C D^{\prime}}$ respectively. In order to evaluate how well $C D^{\prime}$ resembles $C D$, conceptual equivalence will cross-exam how well $C D$ 's data support Concept $_{C D^{\prime}}$ and how well $C D^{\prime}$ 's data support Concept $_{C D}$. The better both data support each other's concept, the less noise in $C D^{\prime}$ and the higher the conceptual equivalence. The process is illustrated in Figure 3.

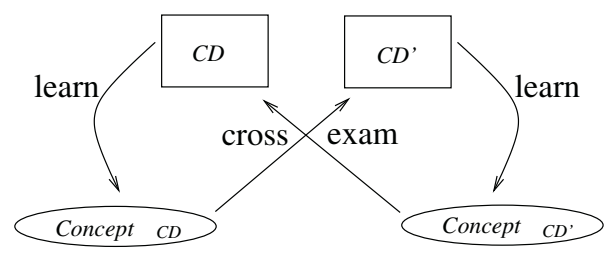

Fig. 3. Conceptual equivalence conducts cross-exam.

\section{Experiments}

Experiments are conducted to test three hypotheses for unpredictable attributes: (1) our new strategy identifies noise more accurately and efficiently than polishing; (2) if the data's genuine concept is learnable and hence cleansing is allowed, our new strategy cleanses noise more effectively than polishing; and (3) conceptual equivalence is more appropriate than literal equivalence to measure noise. Please be noted that we do not claim that our new strategies outperform polishing for predictable attributes. Instead, the niche of our methodology lies within unpredictable attributes.

\subsection{Data and Design}

We choose a dataset from the UCI data repository [1] if it satisfies the following conditions. First, its genuine concept is documented. We do not use this information during any stage of identifying or cleansing. It only helps us check the 
types of attributes and verify our analysis. Second, its genuine concept is learnable, that is, the prediction accuracy is high. Otherwise, we will not be able to differentiate the clean from the noisy. Third, the attributes are predictive but unpredictable. Here we deem an attribute unpredictable if its prediction accuracy is significantly lower than the class. Fourth, there are no numeric attributes. Our approaches can apply to numeric attributes if discretization is employed. However, discretization may introduce extra intractable noise and compromises our understanding of the experimental results. The resulting 5 (3 natural, 2 synthetic) datasets' statistics and prediction accuracies for the class and attributes are in Table 2 .

We use C4.5rules [9] as the rule learner for both noise identification and cleansing. Since originally polishing employs C4.5trees, we re-implement polishing using C4.5rules to ensure a fair comparison. Each attribute is randomly corrupted, where each value other than the original value is equally likely to be chosen. A noise level of $x \%$ indicates that $x \%$ instances are corrupted. Each original dataset is corrupted into four levels: 10\%, 20\%, 30\% and 40\% respectively.

Table 2. Experimental datasets.

\begin{tabular}{l|r|c|c|r|c}
\hline Data & Size & Class & Attribute & \multicolumn{3}{|c}{ Prediction accuracy (\%) } \\
\cline { 4 - 6 } set & & No. & No. & Class & Attributes \\
\hline car & 1728 & 4 & 6 & 92.4 & $29.3,30.7,23.2,46.8,36.6,52.4$ \\
monks1 & 432 & 2 & 6 & 96.5 & $51.2,48.1,41.0,23.1,35.9,44.7$ \\
monks3 & 432 & 2 & 6 & 100.0 & $23.1,51.2,41.2,28.5,35.4,44.2$ \\
ttt $^{a}$ & 958 & 2 & 9 & 85.1 & $51.0,48.9,50.8,49.6,62.7,49.1,49.8,49.3,51.0$ \\
nursery & 12960 & 5 & 8 & 97.1 & $42.8,27.7,24.2,25.5,37.5,50.8,34.4,75.9$ \\
\hline
\end{tabular}

${ }^{a}$ The ttt dataset represents the tic-tac-toe dataset.

\subsection{Identification}

The identification performance is measured by F1 measure [10], a popular measure used in information retrieval that evenly combines precision and recall of the identification. Precision $p$ reflects the purity of identification. It equals the number of truly noisy instances identified divided by the total number of identified instances. Recall $r$ reflects the completeness of identification. It equals to the number of truly noisy instances identified divided by the total number of truly noisy instances. $F 1(p, r)$ equals to $\frac{2 p r}{p+r}$, which falls in the range [0,1]. The higher a method's $F 1$ measure, the better this method simultaneously maximizes both precision and recall. The results for all 20 cases (5 datasets, each with 4 levels of corruption) are depicted in Figure 4. No matter whether one uses matchcorresponding or uses match-anyone ${ }^{4}$, sifting achieves a higher $F 1$ measure in almost all cases. This indicates that sifting outperforms polishing in identifying noise for unpredictable attributes.

\footnotetext{
${ }^{4}$ Conceptual equivalence does not apply here since no data have been cleansed yet.
} 


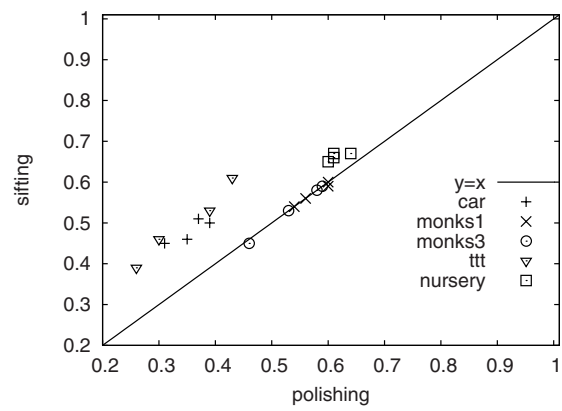

(a) Match-corresponding

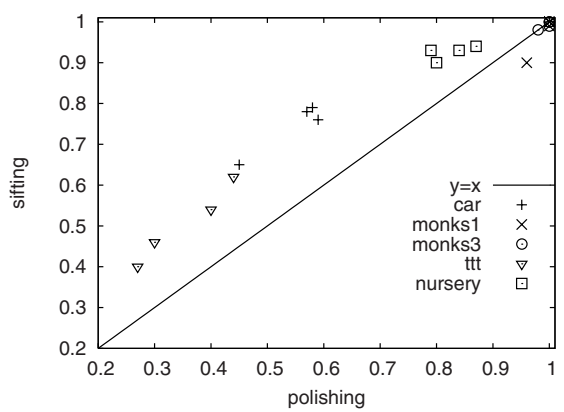

(b) Match-anyone

Fig. 4. The F1 measure measures sifting and polishing's identification. Each coordinate represents an experimental case. Its $x$ component represents polishing's $F 1$ measure and its $y$ component represents sifting's $F 1$ measure. Hence, any coordinate above the $y=x$ line indicates a case where sifting achieves a higher $F 1$ measure than polishing does.

\subsection{Cleansing}

The cleansing performance under literal and conceptual equivalence is studied here.

Under literal equivalence. Figure 5 records correction accuracy of unisubstitution and polishing ${ }^{5}$ in each of 20 cases, which equals to the number of correctly cleansed instances divided by the total number of identified instances. Although uni-substitution achieves higher correction accuracies more often than not, the results also confirm our belief that literal equivalence tends to improperly reflect the cleansing performance. Correction accuracies under match-corresponding are always low (below 10\%) since match-corresponding is sensitive to the instances' order and is over censorious. Correction accuracies under match-anyone are far higher since match-anyone can be insensitive to information loss and has a potential to exaggerate the cleansing efficacy.

However, ttt is an exception that obtains a low accuracy even under matchanyone. Its clean data encode the complete set of legal 3x3 board configurations at the end of tic-tac-toe games and contain 958 instances. But the corruption does not have the legitimacy in mind and has $3^{9}=19683$ configurations at choice. Few can be restored to completely match a clean instance. This raises another type of situation where literal equivalence can not manage well.

Under conceptual equivalence. There can be different ways to calculate conceptual equivalence. Table 3 reports the results of our implementation. We first learn classification rules from clean data and use them to classify

\footnotetext{
${ }^{5}$ Correction accuracy here does not apply to deletion or multi-substitution since they
} do not maintain the original data amount. 


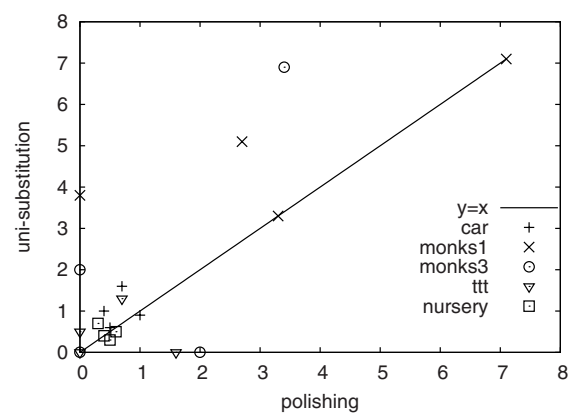

(a) Match-corresponding

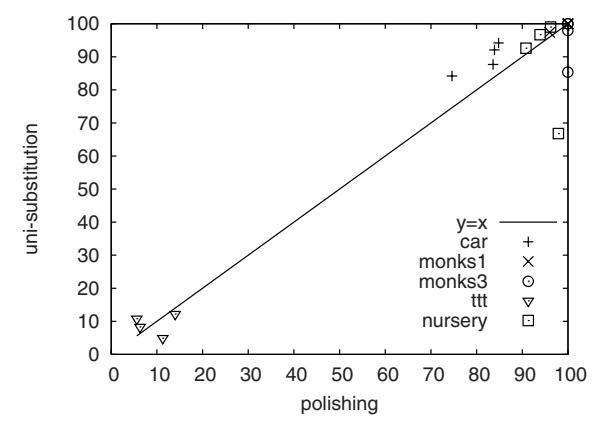

(b) Match-anyone

Fig. 5. Under literal equivalence, the correction accuracy $(\%)$ measures polishing and uni-substitution's cleansing. Each coordinate represents an experimental case. Its $x$ component represents polishing's correction accuracy and its $y$ component represents uni-substitution's correction accuracy. Hence, any coordinate above the $y=x$ line indicates a case where uni-substitution achieves a higher correction accuracy than polishing does. The axis value ranges of (a) and (b) are different. The correction accuracies under match-corresponding are far lower than those under match-anyone.

cleansed data, obtaining a classification accuracy $a c c_{1}$. We then learn classification rules from cleansed data and use them to classify clean data, obtaining another classification accuracy $a c c_{2}$. Because $a c c_{1}$ and $a c c_{2}$ can be associated with different data sizes $\left(\text { size }_{1} \text { and } \text { size }_{2} \text { respectively }\right)^{6}$, their weighted mean $=\sum_{i=1}^{2}\left(a c c_{i} \times s_{i z e_{i}}\right) / \sum_{i=1}^{2}$ size $_{i}$ is used to indicate the degree of conceptual equivalence as in (a). Graphs are drawn in (b) and (c) to better reflect the trend of the values. The pattern in (b) corresponds to 'car', which is representative of other three datasets, monks1, monks2 and nursery. The pattern in (c) corresponds to 'ttt', which is a little different from others because of ttt's special nature as we have explained in the previous section.

Compared with corrupted data, all of deletion, uni-substitution and multisubstitution achieve higher conceptual equivalence across all datasets at all noise levels. This suggests that our handling helps improve the quality of the corrupted data. Compared among themselves, there is no significant difference. An interesting observation is that, despite the risk of losing information, deletion works surprisingly well. A closer look reveals that deletion gains this advantage mainly through large datasets like nursery. This suggests that when the available data well exceed the amount that is needed to learn the underlying concept, appropriately deleting suspicious noise may not harm the genuine reflection of the concept while may effectively eliminate the chance of introducing new noise. As for multi-substitution, we have observed that multiple candidates do not often happen. It is because our process of filtering is very fine as given in Algorithm 2. One can make it coarser and may observe more differences between multisubstitution and uni-substitution.

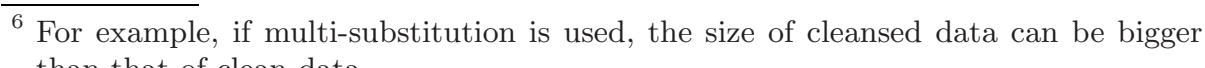
than that of clean data. 
Table 3. Cleansing performance under conceptual equivalence (\%).

\begin{tabular}{r|l|r|r|r|r|r|r}
\hline $\begin{array}{l}\text { Noise } \\
\text { level }\end{array}$ & Data & Cor & Del & Mul & Uni & Pol & Rev \\
\hline \multirow{4}{*}{$\begin{array}{l}\text { set } \\
\text { car }\end{array}$} & 94.8 & 97.1 & 97.6 & 97.8 & 95.6 & 97.0 \\
& monks1 & 98.4 & 100.0 & 100.0 & 100.0 & 100.0 & 100.0 \\
& monks3 & 98.3 & 100.0 & 100.0 & 100.0 & 100.0 & 100.0 \\
& ttt & 97.3 & 98.0 & 97.8 & 97.8 & 98.4 & 99.3 \\
& nursery & 96.6 & 99.3 & 99.2 & 99.2 & 99.0 & 99.1 \\
\hline \multirow{5}{*}{ 20\% } & car & 92.2 & 96.8 & 96.8 & 96.8 & 95.1 & 95.9 \\
& monks1 & 96.6 & 100.0 & 100.0 & 100.0 & 100.0 & 100.0 \\
& monks3 & 96.7 & 100.0 & 100.0 & 100.0 & 100.0 & 100.0 \\
& ttt & 92.9 & 94.3 & 94.1 & 93.4 & 93.1 & 94.7 \\
& nursery & 93.7 & 99.0 & 98.8 & 98.8 & 97.9 & 98.8 \\
\hline \multirow{5}{*}{$30 \%$} & car & 86.9 & 93.9 & 93.0 & 93.0 & 91.6 & 93.9 \\
& monks1 & 93.8 & 100.0 & 100.0 & 100.0 & 100.0 & 100.0 \\
& monks3 & 93.9 & 99.5 & 99.4 & 99.4 & 99.5 & 99.5 \\
& ttt & 87.8 & 89.9 & 88.5 & 88.6 & 87.6 & 89.3 \\
& nursery & 90.4 & 98.5 & 95.5 & 95.6 & 96.9 & 98.0 \\
\hline \multirow{4}{*}{$40 \%$} & car & 87.4 & 91.7 & 92.7 & 92.5 & 89.5 & 91.1 \\
& monks1 & 91.3 & 99.2 & 98.8 & 98.8 & 99.7 & 97.8 \\
& monks3 & 94.4 & 99.9 & 98.4 & 98.4 & 100.0 & 99.9 \\
& ttt & 82.3 & 87.5 & 86.4 & 86.4 & 83.9 & 85.6 \\
nursery & 87.3 & 97.3 & 96.5 & 96.6 & 94.3 & 96.6 \\
\hline Mean & 92.6 & 97.1 & 96.7 & 96.6 & 96.1 & 96.8 \\
Geomean & 92.5 & 97.0 & 96.6 & 96.6 & 96.0 & 96.7 \\
\hline
\end{tabular}

(a) summary

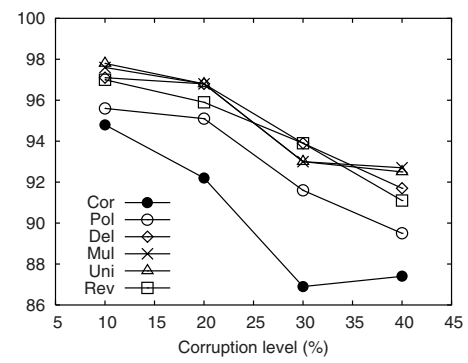

(b) car

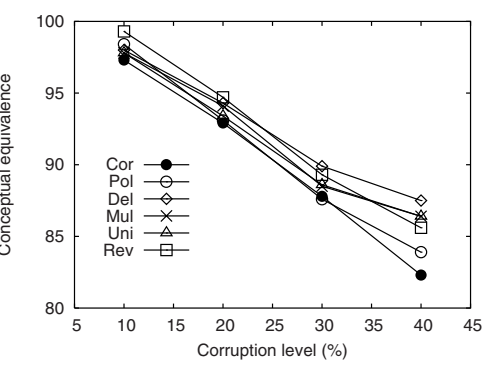

(c) $\mathrm{ttt}$

Note: Each method's conceptual equivalence is calculated between the clean data and the data processed by this method. 'Cor' is corruption; 'Del' is deletion; 'Mul' is multi-substitution; 'Uni' is uni-substitution; 'Pol' is polishing; and 'Rev' is the revised version of polishing that is supplied with sifting's identification. The 'Mean' row and 'Geomean' row record its arithmetic mean and geometric mean across different datasets.

Compared with polishing, all of our cleansing methods achieve higher conceptual equivalence more often than not. Their arithmetic and geometric means are also higher than polishing's. Nevertheless, we do not jump to the conclusion that polishing's cleansing, which is sophisticated, is inferior. It is possible that the disadvantage of its identification is passed on to its cleansing. Hence, we implement a revised version of polishing whose cleansing is supplied with sifting's identification. Thus we can have a pure comparison between the cleansing performances. The experimental results show that revised polishing either improves on polishing or maintains polishing's high conceptual equivalence (like 100\%) in 18 out of 20 cases. This from another perspective verifies that sifting is effective.

The revised polishing thus can obtain competitive conceptual equivalence. However, in terms of efficiency, our new strategies are superior to polishing. Suppose the number of instances and attributes to be $I$ and $A$ respectively. Suppose the rule learning algorithm's time complexity and the number of learned 
rules to be $O(L)$ and $R$ respectively. For each attribute, polishing conducts cross validation to predict its value for each instance. It then recursively tries out different combinations of attribute changes for each instance. It reaches a prohibitive time complexity of $O(A L I)+O\left(I 2^{A}\right)$. In comparison, our cleansing needs to learn a rule set for once and match each instance against this rule set. Thus it has a time complexity of $O(L)+O(I R)$. Hence our methods are far more efficient than polishing, which has been verified by the experimental running time and is important in the real world where large data are routinely involved.

\section{Conclusion}

This paper handles predictive-but-unpredictable attributes in noisy data sources. To identify noise, we have proposed sifting. To cleanse noise, we have suggested that unless the genuine concept can be reliably learned, one should be very cautious to modify an instance. When the genuine concept is learnable, we have studied three cleansing approaches, deletion, uni-substitution and multisubstitution. To measure noise, we have argued that literal equivalence is often inadvisable and proposed conceptual equivalence. Both theoretical analysis and empirical evidence have demonstrated that our strategies achieve better efficacy and efficiency than previous alternatives.

The knowledge acquired by our study, although preliminary, is informative. We expect it to contribute to completing the picture of attribute noise handling. However, a single study seldom settles an issue once and for all. More efforts are needed to further advance this research field. We name three topics here.

First, whether an attribute is predictable or unpredictable is a matter of degree. In our current research, we deem an attribute unpredictable when its prediction accuracy is significantly lower than the class. Further research to work out more sophisticated thresholds or heuristics would be interesting. Second, although we take polishing as straw man, sifting does not claim to outperform polishing for predictable attributes. Instead, sifting and polishing are parallel, each having its own niche to work. Hence it is sensible to combine them. A work frame might be: (1) use feature selection to discard unpredictive attributes; (2) decide whether a predictive attribute is predictable; (3) if it is predictable, use polishing to handle its noise; and if it is unpredictable, use sifting to handle its noise. Lastly, it would be enchanting to extend our research beyond classification learning, such as to association learning where patterns exist but attributes are seldom predictable.

\section{Acknowledgement}

This research has been supported by the U.S. Army Research Laboratory and the U.S. Army Research Office under the grant number DAAD19-02-1-0178. 


\section{References}

1. Blake, C. L., And Merz, C. J. UCI repository of machine learning databases, Department of Information and Computer Science, University of California, Irvine, 1998.

2. Brodley, C. E., And Friedl, M. A. Identifying and eliminating mislabeled training instances. In Proc. of the 13th National Conf. on Artificial Intelligence (1996), pp. 799-805.

3. Brodley, C. E., And Friedu, M. A. Identifying mislabeled training data. Journal of Artificial Intelligence Research 11 (1999), 131-167.

4. Gamberger, D., Lavrac, N., and Dzeroski, S. Noise detection and elimination in data preprocessing: experiments in medical domains. Applied Artificial Intelligence 14 (2000), 205-223.

5. Gamberger, D., Lavrac, N., and Groselu, C. Experiments with noise filtering in a medical domain. In Proc. of the 16th International Conf. on Machine Learning (1999), pp. 143-151.

6. Guyon, I., Matic, N., And VApnik, V. Discovering Informative Patterns and Data Cleaning. AAAI/MIT Press, 1996, pp. 181-203.

7. Kubica, J., And Moore, A. Probabilistic noise identification and data cleaning. In Proc. of the 3rd IEEE International Conf. on Data Mining (2003), pp. 131-138.

8. Maletic, J. I., And Marcus, A. Data cleansing: Beyond integrity analysis. In Proc. of the 5th Conf. on Information Quality (2000), pp. 200-209.

9. Quinlan, J. R. C4.5: Programs for Machine Learning. Morgan Kaufmann Publishers, 1993.

10. Rijsbergen, C. J. V. Information Retrieval, second edition. Butterworths, 1979.

11. Schwarm, S., And Wolfman, S. Cleaning data with Bayesian methods, 2000. Final project report for CSE574, University of Washington.

12. Teng, C. M. Correcting noisy data. In Proc. of the 16th International Conf. on Machine Learning (1999), pp. 239-248.

13. Teng, C. M. Applying noise handling techniques to genomic data: A case study. In Proc. of the 3rd IEEE International Conf. on Data Mining (2003), pp. 743-746.

14. Verbaeten, S. Identifying mislabeled training examples in ILP classification problems. In Proc. of the 12th Belgian-Dutch Conf. on Machine Learning (2002), pp. $1-8$.

15. Zhu, X., Wu, X., And Chen, Q. Eliminating class noise in large datasets. In Proc. of the 20th International Conf. on Machine Learning (2003), pp. 920-927. 